\title{
REVISIÓN
}

\section{Hongos ectomicorrícicos y la tolerancia a la salinidad en plantas}

\author{
Ectomycorrhizal fungi and tolerance to salinity in plants \\ SELENE AGUILAR-AGUILAR ${ }^{1}$, JESÚS PÉREZ-MORENO ${ }^{2}$, RONALD FERRERA-CERRATO $^{2}$, \\ ONÉCIMO GRIMALDO-JUÁREZ ${ }^{1}$, LOURDES CERVANTES-DÍAZ ${ }^{1}$ \& DANIEL GONZÁLEZ-MENDOZA ${ }^{1}$, * \\ ${ }^{1}$ Instituto de Ciencias Agrícolas Universidad Autónoma de Baja California (ICA-UABC) Carretera a Delta s/n, Código \\ Postal 21705, Ejido Nuevo León, Baja California, México \\ ${ }^{2}$ Laboratorio de Microbiología, Edafología-IRENAT, Colegio de Postgraduados, km 36,5 Carretera México-Texcoco, \\ Montecillo, Texcoco, Estado de México, Código Postal 56230, México \\ *e-mail para correspondencia: daniasaf@gmail.com
}

\begin{abstract}
RESUMEN
El proceso de salinización de los suelos constituye un problema generalizado a nivel global. En este sentido, los hongos ectomicorrícicos tienen una importante participación en la recuperación de suelos forestales ya que involucran una serie de mecanismos celulares que pueden contribuir a la tolerancia a la salinidad en plantas que habitan los bosques templados o boreales. La participación de los hongos ectomicorrícicos en la tolerancia a la salinidad involucra la regulación homeostática de los iones, la mejora de captación de agua y la inducción de genes específicos en las raíces colonizadas. Los hongos ectomicorrícicos pueden estimular la presencia de osmolitos como la prolina, azúcares y polioles que contribuyen en la protección de las células vegetales. Además, estos organismos inducen la síntesis de enzimas antioxidantes y glutatión que participan en la disminución de especies reactivas de oxígeno. Esta revisión ofrece una descripción de la participación de los hongos ectomicorrícicos en la tolerancia a la salinidad en plantas.
\end{abstract}

Palabras clave: salinidad, hongos ectomicorrícicos, tolerancia.

\begin{abstract}
The process of salinization of the soil is a widespread problem at the global level. In this sense, ectomycorrhizal fungi have an important role in the recovery of forest soil, as it involves a number of cellular mechanisms that may contribute to the salinity tolerance in plants that inhabit temperate and boreal forests. The participation of ectomycorrhizal fungi on the salinity tolerance involves the ion-homeostasis regulation, improving uptake water and inducing specific gene in roots colonized. Likewise ectomycorrhizal fungi can stimulate the presence of osmolytes as proline, sugars and polyols that contribute to the protection of plant cells. Additionally, these organisms stimulate the synthesis of glutathione and antioxidant enzymes involved in the decrease of reactive oxygen species. This review provides an overview of participation of ectomycorrhizal fungi in the salinity tolerance in plants.
\end{abstract}

Key words: salinity, ectomycorrhizal fungi, tolerance.

\section{INTRODUCCIÓN}

El proceso de salinización del suelo es uno de los principales factores abióticos que limitan la generación de alimentos, debido a que disminuye la capacidad de producción de los campos agrícolas (Flowe 2004). Diversos estudios han demostrado que casi la mitad de zonas agrícolas bajo riego, principalmente de zonas áridas y semiáridas, son afectadas por la salinización del suelo (Munns 2002, Flower 2004). En plantas, concentraciones excesivas de iones como $\mathrm{Na}$ y $\mathrm{Cl}$ en las células pueden generar un estrés osmótico, lo cual puede comprometer la homeostasis celular en las plantas, alterando la integridad de la membrana y ocasionando la muerte celular (Zhu 2002, Langenfeld-Heyser 2007). Debido a esto, las 
plantas han desarrollado a través de su proceso evolutivo una serie de mecanismos externos e internos que involucran diversas adaptaciones anatómicas y morfológicas, así como una compleja red de mecanismos a nivel bioquímico. Entre las estrategias internas que la planta tiene para regular el contenido de iones se puede mencionar: acumulación o exclusión de iones; control de la absorción de los iones por la raíz y su transporte a tejido foliar; compartimentalización en células de la planta; síntesis de solutos compatibles; cambios en la estructura de la membrana e inducción de enzimas antioxidantes y hormonas (Marcum 1999, Bandou et al. 2006). Lo anterior ha permitido clasificar a las plantas en base a la capacidad de crecer en ambientes salinos en glicófitas y halófitas, las primeras se caracterizan por ser altamente sensibles a las variaciones de salinidad; mientras que, por el contrario, las halófitas se caracterizan por ser tolerantes y desarrollarse en ambientes con elevadas concentraciones de sales (e.g., 500 $\mathrm{mM}$ de $\mathrm{NaCl}$ ). En base a lo anteriormente descrito se puede definir el fenómeno de tolerancia a la salinidad en los organismos vegetales como el proceso evolutivo que confiere a distintas especies de plantas la capacidad de crecer y desarrollarse en ambientes con presencia excesiva de electrolitos (Parida \& Das 2005). Las estrategias que la célula vegetal ha desarrollado para mantener el equilibrio homeostático son en general conocidas; no obstante, son pocos los estudios sobre la participación de los hongos ectomicorrícicos (HEM) en la tolerancia a la salinidad en especies de importancia forestal.

Recientemente, los HEM han cobrado interés debido a los programas de reforestación o restauración de suelos mediante el uso de especies forestales, en donde la presencia de HEM es de vital importancia para mantener y estimular la absorción y retención de agua, la fijación de $\mathrm{CO}_{2}$ y contribuir a la recuperación de ecosistemas forestales (Cha-uma et al. 2003).

\section{La simbiosis HEM y plantas forestales}

Los HEM se caracterizan por ser asociaciones mutualistas obligadas entre hongos y raíces de plantas superiores que han evolucionado a través del tiempo (130-180 millones de años), lo cual ha generado una amplia diversificación de los micobiontes, principalmente en los bosques templados y boreales, en donde alrededor del 95 $\%$ de las especies forestales establecen la asociación ectomicorrícica. Los hongos que forman las ectomicorrizas son principalmente Basidiomicetos, aproximadamente unas 5.000 especies, y algunos Ascomicetos que se asocian con unas 3.000 especies diferentes de plantas (García-Rodríguez 2006). El establecimiento de la interacción entre los HEM y las plantas es el resultado de una compleja red de mecanismos a nivel bioquímico que se inicia a través de la germinación de las esporas que producen hifas monocarióticas, las cuales se fusionan y forman el micelio dicariótico que interactúa con el sistema radicular de las plantas. Posteriormente, las hifas se adhieren a la superficie de las raíces formando una estructura conocida como manto fúngico que tiene generalmente un espesor de 20-100 $\mu \mathrm{m}$. En cuanto al intercambio de nutrientes entre ambos organismos se realiza mediante la red de Hartig, que se forma por la penetración de las hifas entre los espacios intercelulares de células epidérmicas en Angiospermas y entre las capas de células corticales en Gimnospermas (Pérez-Moreno \& Read 2004), en donde el micelio que está vinculado con la hifa extramatricial tiene la función de explorar el sustrato facilitando la absorción, transporte de nutrientes y agua al tejido radicular de la planta.

\section{Los HEM y la tolerancia a salinidad en plantas}

Estudios realizados in situ e in vitro han observado que la asociación de los hongos ectomicorrícicos y plantas suele disminuir con el incremento de la salinidad en el suelo o en el medio de crecimiento. No obstante, la tolerancia a la salinidad puede variar entre las especies de los HEM; por ejemplo, se ha observado que géneros como Pisolithus, Laccaria y Suillus son aparentemente más tolerantes a las sales de sodio que Thelephora o Cenococcum. Por otra parte, Reddell et al. (1986) y Dixon (1993) observaron que especies de Frankia y Suillus presentaban procesos de compartimentalización de las sales en vacuolas y en su pared celular, excluyendo a los iones tóxicos de los procesos metabólicos. Por su parte, Chen et al. (2001) observaron en especies 
de Pisolithius (18 aislados) una alta resistencia al $\mathrm{NaCl}$ en suelos salinos. Lo cual sugiere la presencia de un eficiente proceso de osmorregulación en el citoplasma, que mantiene una alta actividad metabólica y mantiene los potenciales de agua para evitar el marchitamiento de la planta. Recientemente, en los programas de reforestación de sitios salinos ha cobrado interés el uso de los HEM, lo cual ha motivado investigaciones enfocadas a identificar y aislar cepas de HEM tolerantes a salinidad.

Estudios recientes indican que los HEM pueden tener diferencias en el grado de tolerancia a la salinidad. Por ejemplo, al exponer diferentes cepas de Paxillus involutus (Batsch ex Fr.) Fr y Pisolithus tinctorius (Mich.: Pers.) Coker \& Couch a $\mathrm{NaCl}(500$ $\mathrm{mM}$ ) se ha observado la presencia de variaciones intraespecíficas en la tolerancia (Matsuda et al. 2006, Langenfeld-Heyser et al. 2007). Por otra parte, se ha observado que plantas de Picea glauca (MoenchVoss) y Pinus banksiana Lamb, inoculadas con Hebeloma crustuliniforme (Bull) Quel. UAMH 5247, Laccaria bicolor Maire (Orton) UAMH 8232 y Suillus tomentosus (Kauff.) Sing., mostraron un incremento del $100 \%$ en su sobrevivencia al crecer en zonas impactadas por $\mathrm{NaCl}$ (Bois et al. 2006). Lo cual ha incrementado el interés en conocer los mecanismos de tolerancia a salinidad que son inducidos por los HEM en las plantas, mediante el empleo de herramientas fisiológicas, bioquímicas y moleculares. Esto con la finalidad de generar estrategias que permitan hacer más eficientes los programas de reforestación de los suelos con elevado contenido de sales.

Mecanismos de exclusión y acumulación en los HEM

Entre los mecanismos que los HEM presentan para proteger a las plantas del efecto tóxico de los iones se pueden mencionar los de exclusión y compartimentalización. Estos procesos actúan como una primera línea de defensa en donde las estructuras de los HEM funcionan como extensiones de las raíces que retienen los iones principalmente en vacuolas, sin afectar sus funciones metabólicas. Este proceso tiene lugar principalmente en las hifas externas y micelio de los HEM en donde la entrada de iones a la planta es regulada por estas estructuras. El modo de acción de este mecanismo involucra de manera general mantener altas concentraciones de $\mathrm{K}$ y baja concentraciones de $\mathrm{Na}$ en el citosol en las células del HEM. Lo anterior es posible debido a la expresión y actividad de transportadores de $\mathrm{Na}$ y $\mathrm{K}$; así como de bombas de $\mathrm{H}^{+}$que regulan el transporte de los iones en las células del HEM (Corratgé et al. 2007). Esto ha sido recientemente descrito por Langenfeld-Heyser et al. (2007), quienes registraron una disminución en el flujo del $\mathrm{Na}$ y un incremento de K a través del xilema en plantas de Populus canescens (Ait.) Sm. (pro sp.) inoculadas con Paxillus involutus. Por otra parte, Bandou et al. (2006) observaron que al exponer plantas de Coccoloba uvifera L., micorrizadas con Scleroderma bermudense Coker a diferentes dosis de $\mathrm{NaCl}$, estas presentaron menor presencia a iones en sus raíces reflejándose en un mejor estado fisiológico con respecto a las plantas no micorrizadas.

\section{Mejora en la economía de nutrientes}

Los HEM también contribuyen a disminuir el efecto que tiene la salinidad en la movilidad y absorción de nutrientes, en las raíces de las plantas mediante una mejora en la captación de nutrientes y agua. Esto debido a que la red de hifas facilita la absorción de nutrientes como $\mathrm{P}$ y $K$ ya que existe una mayor área de exploración del sustrato (Munn \& Mosse 1980, Giri \& Mukerji 2004, Tain et al. 2004).

Trabajos realizados por Plamboeck et al. (2007) muestran que la red de hifas participa activamente en el transporte del agua hacia la planta. En este sentido, investigaciones realizadas por Tagu et al. (1996) y Bücking et al. (2002) en plantas de Eucalyptus globulus Labill micorrizadas con Pisolithus tinctorius muestran la expresión de genes que sintetizan pequeñas proteínas hidrofóbicas, llamadas hidrofóbinas, que participan en la agregación de la hifa del HEM. La función principal de estas proteínas no se conoce con exactitud, pero se sabe que evitan la deshidratación del manto hifal y generan una barrera que limita la difusión de elementos como $\mathrm{Ca}, \mathrm{Mg}$ y $\mathrm{K}$ a la raíz micorrizada, contribuyendo a la disminución del estrés salino (Mankel et al. 2002). 
No obstante, aun cuando se han demostrado los efectos protectores de los HEM en la disminución de la absorción de sales por la raíz y la mejora en el suministro de nutrientes hacia la planta bajo estrés salino, existe una serie de variables biológicas y ambientales que deben ser consideradas en la selección de cepas de HEM. Entre estos factores se pueden mencionar la especie de planta, la especificidad de la cepa de hongo ectomicorrícico, las condiciones edáficas y el manejo del cultivo.

\section{Presencia de osmoprotectores en los HEM}

Entre las sustancias osmoprotectoras que pueden ser estimuladas en los HEM, se puede mencionar a los polioles, estos se caracterizan por ser formas reducidas de monosacáridos y pueden clasificarse en acíclicos (e.g., manitol) y cíclicos (e.g., pinitol). Los HEM pueden sintetizar y acumular solutos compatibles u osmoprotectores en el citoplasma, para mantener el equilibrio homeostático en el interior de las células del hongo y proteger al sistema radical colonizado de la planta (Noiraud et al. 2001). Entre las funciones fisiológicas que tienen los polioles se puede mencionar su participación en la osmoprotección de plantas; así como en la disminución de especies reactivas de oxígeno (Parida et al. 2007). Entre los polioles, que han sido evaluados en los HEM, se puede citar al manitol, que ha sido observado principalmente en hongos Basidiomicetos, expuestos a diferentes dosis de salinidad. En donde su función es la de actuar como un soluto compatible contribuyendo a disminuir el estrés salino en las células del HEM y evitar el daño de las raíces colonizadas de las plantas (Bois et al. 2006). Por otra parte, la presencia de otros osmoprotectores como la prolina también ha sido evaluada en los HEM. La prolina es un aminoácido que en condiciones de estrés salino aumenta para actuar como un agente osmótico, protegiendo a las células del estrés salino. Esto debido a que la prolina actúa como un mediador del ajuste osmótico ya que estabiliza a las proteínas y membranas, además de inducir genes relacionados con el estrés salino (Khedr et al. 2003). Lo anterior fue observado en HEM de las especies Hymenoscyphus spp., que al ser expuestos a distintas dosis de $\mathrm{NaCl}$ se incrementaba el contenido de prolina que contribuía a evitar un déficit de agua en la planta expuesta al estrés salino (Bois et al. 2006).

Asimismo, se ha reportado en plantas micorrizadas de Nothofagus dombeyi (Mirb.) Oerst., un incremento de prolina que genera un mejor ajuste osmótico, que contribuye a la funcionalidad del aparato fotosintético de la planta (Alberdi et al. 2007).

Por otra parte, Shi et al. (2002) mencionan que los HEM tienen una posible participación regulando el contenido de azúcares (e.g., trealosa), contribuyendo a la protección de la integridad celular de plantas de Fagus sylvatica Linneo al estar expuesta a $\mathrm{NaCl}$ debido a que la trealosa actúa como osmoprotector y secuestrador de especies reactivas de oxígeno en las células de las raíces de plantas micorrizadas (Ferreira et al. 2007).

\section{Enzimas antioxidantes}

El estrés ocasionado por los iones $\mathrm{Na}$ y $\mathrm{Cl}$ en las plantas puede generar un déficit en la absorción de agua que afecta las actividades metabólicas de las células, y genera la presencia de especies reactivas de oxígeno como superóxido, peróxido de hidrógeno y radicales hidroxilo (ERO), los cuales pueden ocasionar severos daños a la integridad de la membrana celular a través de procesos de lipoperoxidación; así como afectar la integridad de ácidos nucleicos y proteínas (GonzálezMendoza \& Zapata-Pérez 2008). La presencia de especies reactivas de oxígeno durante el inicio de la simbiosis de HEM y plantas, generalmente son producidas en concentraciones no tóxicas al inicio del proceso de reconocimiento, por lo que sus niveles suelen estar incrementados en las primeras fases de colonización de la raíz por los HEM (Baptista et al. 2007). Lo anterior permite que la simbiosis ectomicorrícica pueda generar una respuesta más rápida en la biosíntesis de novo de enzimas antioxidantes para contrarrestar el efecto negativo generado por el incremento de ERO en plantas cuando está bajo estrés causado por la presencia de $\mathrm{NaCl}$. Lo anterior ha sido reportado por Langenfeld-Heyser et al. (2007), quienes encontraron que plantas de Populus canescens, expuestas a $\mathrm{NaCl}(500 \mathrm{mM})$ y micorrizadas con Paxillus involutus (cepa MAJ), estimularon un incremento mayor en la 
síntesis de enzimas antioxidantes principalmente de peroxidasa que esta relacionado con una disminución del peróxido de hidrógeno y una mayor lignificación de las paredes celulares de la raíz. Por otra parte, estudios realizados por Courbot et al. (2004) en Paxillus involutus, demostraron la presencia de otros agentes antioxidantes, como el glutatión (molécula rica en tioles) que puede actuar como agente antioxidante, al reaccionar con las especies reactivas de oxígeno y formar glutatión oxidado.

\section{Estudios moleculares en HEM y la tolerancia a salinidad en plantas}

La aplicación de herramientas moleculares y bioquímicas en el estudio de la tolerancia a la salinidad en los HEM es reciente y se ha observado que está constituida de una compleja red de diferentes mecanismos que son regulados por grupos de genes que inducen la síntesis de proteínas con funciones específicas. Lo anterior ha sido discutido por Liang et al. (2007), quienes al evaluar la repuesta de Boletus en condiciones de estrés salino mediante el uso de técnicas bioquímicas identificaron diversas proteínas que tienen una función de protección en los HEM. Por otra parte, trabajos realizados por Marjanovic et al. (2005) identificaron la expresión de genes relacionados con acuaporinas (proteínas de canales de agua) en la asociación ectomicorrícica, en donde la expresión de estos genes contribuía a generar un mayor incremento del transporte de agua en la planta y una reducción del impacto negativo de la salinidad. Finalmente, mediante el uso de herramientas bioquímicas y moleculares, es posible evaluar con más detalle los diferentes mecanismos de tolerancia que los hongos ectomicorrícicos han desarrollado para sobrevivir en ambientes con elevadas concentraciones de sales.

\section{CONCLUSIONES}

Los hongos ectomicorrícicos son organismos que proporcionan amplios beneficios a la planta, debido a una compleja red de mecanismos que le confieren la capacidad de suministrar nutrientes y crecer en ambientes salinos. El mejor entendimiento del establecimiento de la simbiosis entre HEM y plantas permitirá establecer procesos biotecnológicos enfocados a la producción y propagación de cepas de HEM y su aplicación en la recuperación de sitios salinos sódicos. La presencia de un mecanismo u otro en la planta colonizada por los HEM no es del todo comprendido, por lo que es necesario realizar futuros estudios que involucren la aplicación de herramientas moleculares y bioquímicas, así como de plantas modelo con el fin de entender con mayor claridad el modo de acción de los mecanismos de tolerancia que ocurren en la simbiosis ectomicorrícica.

\section{AGRADECIMIENTOS}

Se agradece el apoyo al Programa de Mejoramiento del Profesorado (PROMEP), por los apoyos recibos en la Convocatoria de Incorporación de Nuevos Profesores de Tiempo Completo.

\section{LITERATURA CITADA}

ALBERDI M, M ÁLVAREZ, E VALENZUELA, R GODOY, E OLIVARES \& M BARRIENTOS (2007) Response to water deficit of Nothofagus dombeyi plants inoculated with a specific (Descolea antarctica Sing) and non-specific (Pisolithus tinctorious (Pers.) Coker \& Couch) ectomycorrhizal fungi. Revista Chilena de Historia Natural 80: 479491

BANDOU E, F LEBAILLY, F MULLER, M DULORMNE, A TORIBIO, J CHABROL, R COURTECUISSE, C PLENCHETTE, Y PRIN, R DUPONNOIS, M THIAO, S SYLLA, B DREYFUS \& AMBÂ (2006) The ectomycorrhizal fungus Scleroderma bermudense alleviates salt stress in seagrape (Coccoloba uvifera L.) seedlings. Mycorrhiza 16: 559-565.

BAPTISTA P, A MARTINS, MS PAIS, RM TAVARES \& T LINO-NETO (2007) Involvement of reactive oxygen species during early stages of ectomycorrhiza establishment between, Castanea sativa and Pisolithus tinctorius. Mycorrhiza 17: 185-193.

BOIS G, A BERTRAND, Y PICHÉ, MYP FUNG \& DP KHASA (2006) Ectomycorrhizal fungi affect the physiological response of Picea glauca and Pinus banksiana seedlings exposed to a $\mathrm{NaCl}$ gradient. Tree Physiology 26: 1185-1196.

BÜCKING H, AJ KUHN, WH SCHRÖDER \& W HEYSER (2002) The fungal sheath of ectomycorrhizal pine roots an apoplastic barrier for the entry of calcium, magnesium, and potassium into the root cortex? Journal of Experimental Botany 53: 1659-1669. 
DIXON RK, MV RAO \& VK GARG (1993) Salt stress affects in vitro growth and in situ symbioses of ectomycorrhizal fungi. Mycorrhiza 3: 63-68.

CHA-UMA S, K MOSALEEYANON, K SUPAIBULWATANA \& C KIRDMANEE (2004) Physiological responses of Thai neem (Azadirachta siamensis Val.) to salt stress for salt-tolerance screening program. Science Asia 30: 17-23.

CHEN DM, S ELLUL, K HERDMAN \& JWG CAIRNEY (2001) Influence of salinity on biomass production by Australian Pisolithus spp. Isolates. Mycorrhiza 11: 231-236.

CORRATGÉ C, S ZIMMERMANN, R LAMBILLIOTTE, C PLASSARD, R MARMEISSE, J-B THIBAUD, B LACOMBE \& H SENTENAC (2007) Molecular and functional characterization of a $\mathrm{Na}^{+}-\mathrm{K}^{+}$ transporter from the Trk family in the ectomycorrhizal fungus Hebeloma cylindrosporum. Journal of Biological Chemistry 282: 26057-26066.

COURBOT M, L DIEZ, R RUOTOLO, M CHALOT \& P LEROY (2004) Cadmium-responsive thiols in the ectomycorrhizal fungus Paxillus involutus. Applied and Environmental Microbiology 70: 7413-7417.

FERREIRA AS, MR TOTOLA \& AC BORGES (2007) Physiological implications of trehalose in the ectomycorrhizal fungus Pisolithus sp. under thermal stress. Journal of Thermal Biology 32: 34-41.

FLOWERS TJ (2004) Improving crop salt tolerance. Journal Experimental Botany 55: 307-319.

GARCÍA-RODRÍGUEZ JL, J PÉREZ-MORENO, A ALDRETE, VM CETINA-ALCALÁ \& H VAQUERA-HUERTA (2006) Characterization of the wild ectomycorrhizal fungus Pisolithus tinctorius (Pers.) Coker et Couch in culture and in symbiosis with eucalypt and pine. Agrociencia 40: 665-676.

GIRI B \& K MUKERJI (2004) Mycorrhizal inoculant alleviates salt stress in Sesbania aegyptiaca and Sesbania grandiflora under field conditions: evidence for reduced sodium and improved magnesium uptake. Mycorrhiza 14: 307-312.

GONZÁLEZ-MENDOZA D \& O ZAPATA-PÉREZ (2008) Mecanismos de tolerancia a elementos potencialmente tóxicos en plantas. Boletín de la Sociedad Botánica de México 82: 41-49.

KHEDR AHA, MA ABBAS, AA ABDEL WAHID, WP QUICK \& GM ABOGADALLAH (2003) Proline induces the expression of salt-stress-responsive proteins and may improve the adaptation of Pancratium maritimum L. to salt-stress. Journal of Experimental Botany 54: 2553-2562.

LANGENFELD-HEYSER R, J GAO, T DUCIC, TACHD, CF LU, E FRITZ, A GAFUR \& A POLLE (2007) Paxillus involutus mycorrhiza attenuate $\mathrm{NaCl}$-stress responses in the salt-sensitive hybrid poplar Populus canescens. Mycorrhiza 17: 121-131.

LIANG Y, H CHEN, MJ TANG \& SH SHEN (2007) Proteome analysis of an ectomycorrhizal fungus Boletus edulis under salt shock. Mycological Research 111: 939-946.

MANKEL A, K KRAUSE \& E KOT (2002) Identification of a hydrophobin gene that is developmentally regulated in the ectomycorrhizal fungus Tricholoma terreum. Applied and Environmental Microbiology 68: 1408-1413.

MARCUM K (1999) Salinity tolerance mechanisms of grasses in the subfamily Chloridoideae. Crop Science 39: 1153-1160.

MARJANOVIC Z, N UEHLEIN, R KAHLENHOFF, JJ ZWIAZEK, M WEIß, R HAMPP \& U NEHLS (2005) Aquaporins in poplar: what a difference a symbiont makes! Planta 222: 258-268.

MATSUDA Y, F SUGIYAMA, K NAKANISHI \& S ITO (2006) Effects of sodium chloride on growth of ectomycorrhizal fungal isolates in culture. Mycoscience 47: 212-217.

MUNN DN \& B MOSSE (1980) Mineral nutrition of legume crops. En: Summerfield RJ \& AH Bunting (eds) Advances in legume science: 115-125. H.M.S.O., London, United Kingdom.

NOIRAUD N, L MAUROUSSET \& R LEMOINE (2001) Transport of polyols in higher plants. Plant Physiology and Biochemistry 39: 717-728.

REDDELL P, RC FOSTER \& GD BOWEN (1986) The effects of sodium chloride on growth and nitrogen fixation in Casuarina obesa Miq. New Phytologist 102: 397-408

PARIDA AK, VS DAGAONKAR, MS PHALAK, GV UMALKAR \& LP AURANGABADKAR (2007) Alterations in photosynthetic pigments, protein and osmotic components in cotton genotypes subjected to short-term drought stress followed by recovery. Plant Biotechnology Reports 1: 1-37.

PARIDA AK \& AB DAS (2005) Salt tolerance and salinity effects on plants: a review. Ecotoxicology and Environmental Safety 60: 324-349.

PÉREZ-MORENO J \& DJ READ (2004) Los hongos ectomicorrízicos, lazos vivientes que conectan y nutren a los árboles en la naturaleza. Interciencia 29: 239-247.

PLAMBOECK AH, TE DAWSON, LM EGERTONWARBURTON, M NORTH, TD BRUNS \& JI QUEREJETA (2007) Water transfer via ectomycorrhizal fungal hyphae to conifer seedlings. Mycorrhiza 17: 439-447.

SHI LB, M GUTTENBERGER, I KOTTKE \& R HAMPP (2002) The effect of drought on mycorrhizas of beech (Fagus sylvatica L.): changes in community structure, and the content of carbohydrates and nitrogen storage bodies of the fungi. Mycorrhiza 12: 303-311.

TAGU D, B NASSE \& F MARTIN (1996) Cloning and characterization of hydrophobins-encoding cDNAs from the ectomycorrhizal Basidiomycete Pisolithus tinctorius. Gene 168: 93-97.

TAIN CY, G FENG LI XL \& ZHANG FS (2004) Different effects of arbuscular mycorrhizal fungal isolates from saline or non-saline soil on salinity tolerance of plants. Applied Soil Ecology 26: 143-148.

ZHU JK (2002) Salt and drought stress signal transduction in plants. Annual Review of Plant Biology 53: $247-$ 273.

Editor Asociado: Bernardo González

Recibido el 15 de junio de 2008; aceptado el 22 de diciembre de 2008 